\title{
Certification, adaptivity, and reduction in non-intrusive model couplings
}

\author{
Alexandre Verwée* and Ludovic Chamoin*,† \\ * Université Paris-Saclay, ENS Paris-Saclay, CNRS, LMT \\ 4, avenue des Sciences, 91190 Gif-sur-Yvette, France \\ E-mail : alexandre.verwee@ens-paris-saclay.fr \\ E-mail : ludovic.chamoin@ens-paris-saclay.fr \\ ${ }^{\dagger}$ Institut Universitaire de France (IUF)
}

\begin{abstract}
The non-intrusive coupling method [1] is a local analysis method that aims to separate the macroscopic and local scales both from mesh, operator, and loading viewpoints. This method has demonstrated that it can be used for a large set of problems such as those with local plasticity, and it has been implemented in an industrial code. In order to reduce the complexity of a given problem, a coarse model is defined on the total domain with its properties (geometry, connectivity, operator, solver) and a separated fine model is set locally with its different properties. The solution is obtained iteratively by exchanging data on the interface between the global and local models.
\end{abstract}

However, the issue of certification and optimal driving of such a coupling method has been addressed in very few works until now [2]. In the present work, we tackle this subject using the concept of constitutive relation error (CRE) [3] which is a generic tool able to certify the quality of a numerical solution. The main goal is to control the solution of the non-intrusive global-local algorithm, in terms of global error or error in specific quantities of interest, and to optimally drive the coupling algorithm according to quantitative error indicators on individual error sources (discretization, modelling, iterative scheme).

In this context, we will also study the coupling between a global solution raised from the well-known Finite Element Method and a local solution evaluated on-line from a virtual chart [4] constructed offline from a reduced order model in the local area of interest. Such a virtual chart is based on the PGD technique and integrates as variables (i.e. extra-parameters) some features of the local model such as boundary conditions, geometry, or material behaviour [5]. Complementing the non-intrusive globallocal coupling with local ROM leads to a more flexible exchange between interface quantities, and higher performance in terms of computational efficiency and stability.

The talk will start with an overview of the methodology of non-intrusive coupling methods, before presenting the error estimation procedure using the CRE concept and its decomposition into various source contributions for adaptive control. Eventually, the construction and the performance of ROM in the non-intrusive coupling method will be studied and discussed.

\section{REFERENCES}

[1] L. Gendre, 0. Allix, P. Gosselet, F. Comte, Non-intrusive and exact global/local techniques for structural problems with local plasticity, Computational Mechanics, 44:2, 2009

[2] M. Tirvaudey, L. Chamoin, R. Bouclier, J.-C.Passieux, A posteriori error estimation and adaptivity in non-intrusive couplings between concurrent models, Computer Methods in Applied Mechanics and Engineering, vol. 367, 2020

[3] L. Chamoin, P. Dièz, Verifying calculations, forty years on: an overview of classical verification techniques for FEM simulations, SpringerBriefs, 2016

[4] P.E. Allier, L. Chamoin, P. Ladevèze, Proper Generalized Decomposition computational methods on a benchmark problem: introducing a new strategy based on Constitutive Relation Error minimization, Advanced Modeling and Simulation in Engineering Sciences, 2:17, 2015

[5] D. Canales, A. Leygue, F. Chinesta, D. González, E. Cueto, E. Feulvarch, J.-M. Bergheau, and A. Huerta, Vademecum-based GFEM (V-GFEM): optimal enrichment for transient problems, International Journal for Numerical Methods in Engineering, 108:9, 2016 